\title{
Predictors of ADHD persistence from childhood to adulthood
}

Alves M. MD ${ }^{1}$, Oliveira I. MD ${ }^{1}$, Mata M. MD ${ }^{1}$, Marques C. MD ${ }^{1}$

${ }^{1}$ Centro Hospitalar Universitário Lisboa Central, Child and Adolescent Psychiatry Department, Lisbon, Portugal

Head of Department - Pedro Caldeira da Silva, MD
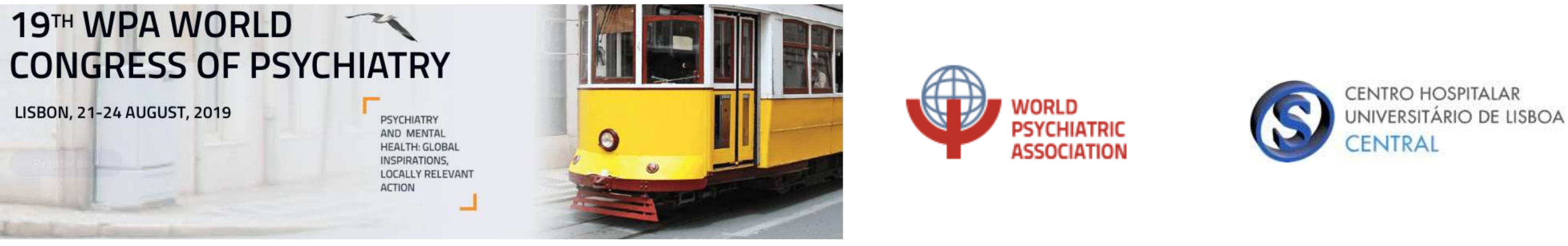

\section{Background and aims:}

Attention-deficit hyperactivity disorder (ADHD) is a common neurodevelopmental disorder that usually persists into adulthood (50\%-70\%). ADHD has extensive comorbidity in childhood and adulthood and a highly variable trajectory. It is important to identify individuals with increased risk of persistence that may benefit from more intensive strategies.

The present study aims to provide an overview of the literature regarding factors that predict the persistence of ADHD from childhood into adulthood.

\section{Methods:}

A nonsystematic review of the literature was performed using the PubMed database searching the terms: "Attention-deficit/hyperactivity disorder", "persistence", "trajectories", "risk markers" and "adulthood".

\section{Results:}

Some predictors were significantly associated with persistence of symptoms into adulthood such as

\begin{tabular}{c|l|} 
Other & - Initial ADHD symptom \\
studies & severity \\
show & $\cdot$ Parental mental health \\
that & $\cdot$ Childhood comorbidity
\end{tabular}

affect persistence of ADHD symptoms into adulthood.

Oppositional defiant disorder and social phobia were associated with the maintenance of symptoms.

Alcohol use disorder $\Rightarrow$ maintenance and rise of symptoms

Some studies found:

- Socio-economic status as potential moderators for - Childhood IQ the prognosis of ADHD
Severe presentation of ADHD

- Major depressive disorder in childhood

\section{Discussion:}

Current knowledge about risk markers of the course of ADHD is still insufficient to guide clinical practice.
Predictors of ADHD perseverance may change with age.
Prevalent comorbidities influence neurodevelopment and the trajectory of ADHD.

\begin{tabular}{c} 
for persistence is essential. \\
\hline Accurate prognosis allows the \\
development of target interventions \\
in childhood and adolescence.
\end{tabular}

Preventive strategies could also play an important role.
Mental health services for children, young people and adults

should develop systems of communication and protocols for information sharing and adequate transition to adult services.

\section{Conclusion:}

Reliable prediction of ADHD persistence or development of comorbidity can change treatment over the the lifespan. Longitudinal studies are essential to chart the trajectory of ADHD symptoms. More studies are needed to identify Risk markers of ADHD persistence or remission in order to better understand the natural history of the disorder. It is important to have more studies that provide valuable insight into ADHD across the lifespan in order to improve medical care. 